\title{
Identification of differentially expressed microRNAs and the potential of microRNA-455-3p as a novel prognostic biomarker in glioma
}

\author{
WEI WANG $^{1 *}$, SHUWEN MU $^{2 *}$, QINGSHUANG ZHAO ${ }^{3}$, LIANG XUE $^{3}$ and SHOUSEN WANG ${ }^{1,3}$ \\ ${ }^{1}$ Department of Neurosurgery, Fuzong Clinical Medical College of Fujian Medical University; \\ ${ }^{2}$ Department of Neurosurgery, Dongfang Affiliated Hospital of Xiamen University; ${ }^{3}$ Department of Neurosurgery, \\ 900 Hospital of The Joint Logistics Team, Fuzhou, Fujian 350025, P.R. China
}

Received April 25, 2019; Accepted September 9, 2019

DOI: $10.3892 / \mathrm{ol} .2019 .10927$

\begin{abstract}
Glioma is an aggressive central nervous system malignancy. MicroRNAs (miRNAs/miRs) have been reported to be involved in the tumorigenesis of numerous types of cancer, including glioma. The present study aimed to identify the differentially expressed miRNAs in glioma, and further explore the clinical value of miR-455-3p in patients with glioma. GEO2R was used for the identification of the differentially expressed miRNAs according to the miRNA expression profiles obtained from the Gene Expression Omnibus database. OncomiR was used to analyze the relationship of miRNAs with the survival outcomes of the patients with glioma. A total of 108 patients with glioma were recruited to examine the expression levels of miR-455-3p and further explore its clinical value. The bioinformatics analysis results suggested that a total of 64 and 48 differentially expressed miRNAs were identified in the GSE90603 and GSE103229 datasets, respectively. There were 12 miRNAs in the overlap of the two datasets, of which three were able to accurately predict overall cancer survival, namely hsa-miR-7-5p, hsa-miR-21-3p and hsa-miR-455-3p. In patients with glioma, miR-455-3p was determined to be significantly upregulated $(\mathrm{P}<0.001)$. Additionally, patients with high miR-455-3p expression had significantly lower 5-year overall survival than those with low miR-455-3p expression (log-rank
\end{abstract}

Correspondence to: Dr Liang Xue, Department of Neurosurgery, 900 Hospital of The Joint Logistics Team, 156 Xi'erhuanbei Road, Fuzhou, Fujian 350025, P.R. China

E-mail: bingni4490552@163.com

Dr Shousen Wang, Department of Neurosurgery, Fuzong Clinical Medical College of Fujian Medical University, 156 Xi'erhuanbei Road, Fuzhou, Fujian 350025, P.R. China

E-mail:wangrumifz@163.com

*Contributed equally

Key words: Gene Expression Omnibus, microRNA-455-3p, prognosis, glioma test, $\mathrm{P}=0.001)$. Cox regression analysis further determined that miR-455-3p was an independent prognostic indicator for overall survival in patients with glioma (hazard ratio $=2.136$; 95\% $\mathrm{CI}=1.177-3.877 ; \mathrm{P}=0.013)$. In conclusion, the present study revealed a series of miRNAs with potential functional roles in the pathogenesis of glioma, and provides findings that indicate miR-455-3p as a promising biomarker for the prognosis of glioma.

\section{Introduction}

Glioma is an aggressive brain cancer with high morbidity and mortality rates (1) characterized by aggressive proliferation, invasion and dismal prognosis (2). Although great progress has been made in the therapeutic field, the prognosis of patients with glioma remains poor (3). Recent studies have revealed a number of aberrantly expressed genes, which may be involved in the progression of glioma $(4,5)$. These findings could provide a new perspective for the development of novel therapeutic strategies for glioma.

MicroRNAs (miRNAs/miRs) are a class of small non-coding RNAs with a length of 20-24 nucleotides (6). miRNA dysregulation has been widely reported in numerous human diseases, especially cancer (7). miRNAs regulate the expression of target messenger RNAs through binding to their 3'-untranslated region (8). Several miRNAs have been identified to serve a crucial role in glioma progression and multiple cellular processes, including cell proliferation, differentiation, apoptosis, migration and invasion. For example, Zhang et al (9) indicated that miR-770 expression was markedly lower in human glioma tissues and cell lines, and that miR-770 overexpression suppressed tumor cell proliferation and induced apoptosis via targeting CDK8. Lu et al (10) demonstrated that the upregulation of miR-6807-3p facilitated carcinogenesis in human glioma through promoting cancer cell proliferation and migration by targeting dachshund homolog 1 . Downregulation of miR-139-3p expression was also detected in glioma tissues, which may be involved in controlling behaviors associated with the malignant progression of glioma, including cell proliferation, migration and invasiveness (11). Thus, there is a great interest in using aberrantly expressed miRNAs as 
prognosis prediction markers for glioma. However, it may be challenging to detect miRNAs involved in glioma occurrence and progression.

In the present study, a number of differentially expressed miRNAs in glioma were detected via bioinformatics analysis. OncomiR analysis suggested that three miRNAs were able to accurately predict overall cancer survival in glioma. The differential expression level of miR-455-3p was found to be the most significant. Its clinical value has not been investigated. Therefore, miR-455-3p levels were further validated in patients with glioma, and its clinical value was explored.

\section{Materials and methods}

Microarray data. Firstly, datasets focusing on miRNA expression were searched using the following search terms: 'Non-coding RNA profiling by array AND glioblastoma' in the National Center for Biotechnology Information Gene Expression Omnibus (GEO) database (https://www.ncbi.nlm. nih.gov/gds/), and only miRNA gene expression profiles were included. Additionally, only datasets based on the comparison of glioma tissues and healthy samples were included. Datasets based on glioma serum, glioma cell lines or animal models were excluded. The GSE90603 and GSE103229 $(12,13)$ datasets met the inclusion criteria and were subsequently assessed using GEO2R (http://www.ncbi.nlm.nih.gov/geo/geo2r) for differential genetic analysis. The combination of the two datasets has not been mentioned in previous studies. GSE90603 contained 16 patients with glioblastoma (GBM) and 7 healthy individuals, while GSE103229 contained 5 GBM samples and 5 normal samples.

Identification of differentially expressed miRNAs. GEO2R (http://www.ncbi.nlm.nih.gov/geo/info/geo2r.html) is an online tool used to compare two or more groups of samples in GEO series for the identification of differentially expressed miRNAs under different experimental conditions. In the present study, GEO2R was used for the identification of differentially expressed miRNAs in glioma, and the adjusted P-value $<0.05$ and $\mid \log$ fold change $(\mathrm{FC}) \mid>2$ were chosen as cut-off levels.

miRNA expression-based survival signature analysis. OncomiR is able to analyze miRNA-derived survival outcome signatures dynamically for one or more types of cancer (14). In the present study, the glioma patients were divided into a high-risk and low-risk cohort according to the expression level of the selected miRNAs, overall cancer survival rates were predicted using OncomiR, which was presented with a Kaplan-Meier survival curve.

Patients and sample collection. A total of 108 patients with primary glioma who underwent primary tumor resection between June 2010 and March 2011 at The 900 Hospital of the Joint Logistics Team were recruited in the present study. The clinicopathological features of glioma patients were collected, including age, sex, tumor size, World Health Organization (WHO) grade and Karnofsky performance scale (KPS). A total of 95 normal brain tissue samples were collected as controls from patients who had undergone surgery for cerebral injury and cerebral hemorrhage at the same hospital. All tissue specimens were immediately snap-frozen in liquid nitrogen and stored at $-80^{\circ} \mathrm{C}$ until further processing. No participants received radiotherapy or chemotherapy prior to surgery. After surgery, all patients were followed up by special clinic reexamination, letters or telephones to calculate the 5-year survival rate. The interval follow-up time was $\sim$ half or one year. All procedures were reviewed and approved by the Ethics Committee of The 900 Hospital of the Joint Logistics Team. Written informed consent was obtained from each participant.

Reverse transcription-quantitative polymerase chain reaction (RT-qPCR) of miR-455-3p. RT-qPCR was used to determine the expression levels of miR-455-3p every two weeks or so since the sample collection. Each experiment was repeated three times. Total RNA was extracted from glioma and normal brain tissues using TRIzol reagent (Thermo Fisher Scientific, Inc.), according to the manufacturer's protocol. The miScript Reverse Transcription Kit (Qiagen) was used for reverse transcription reactions. Next, qPCR was performed using the SYBR Green I Master Mix Kit (Invitrogen; Thermo Fisher Scientific, Inc.) and the 7300 Real-Time PCR System (Applied Biosystems; Thermo Fisher Scientific, Inc.). The primer sequences were as follows: $\mathrm{miR}-455-3 \mathrm{p}$ forward, 5'-GGGGCAGTCCATGGGCAT-3' and reverse, 5'-CTCAAC TGGTGTCGTGGA-3'; and U6 forward, CTCGCTTCGGCA GCACA and reverse, AACGCTTCACGAATTTGCGT. The PCR conditions were as follows: $95^{\circ} \mathrm{C}$ for $5 \mathrm{~min}$, followed by 40 cycles of $95^{\circ} \mathrm{C}$ for $30 \mathrm{sec}, 60^{\circ} \mathrm{C}$ for $30 \mathrm{sec}$, and $72^{\circ} \mathrm{C}$ for $40 \mathrm{sec}$. Relative miR-455-3p expression was normalized to the expression of U6 and was quantified using the $2^{-\Delta \Delta \mathrm{Cq}}$ method (15).

Statistical analysis. SPSS software (version 18.0; SPSS, Inc.) and GraphPad Prism software (version 5.0; GraphPad Software, Inc.) were used for data analysis. Measurement data between groups were evaluated using the Student's t test, whereas counting data were evaluated using the $\chi^{2}$ test. The mean expression level of miR-455-3p in glioma tissues was used as the cutoff, and the patients were divided into low- and high-expression groups. The differences between the two groups were compared using the $\chi^{2}$ test. The overall survival of patients with glioma was assessed using the Kaplan-Meier curve and log-rank test. Cox regression analysis was performed to further assess the prognostic value of miR-455-3p in glioma. $\mathrm{P}<0.05$ was considered to indicate a statistically significant difference.

\section{Results}

Identification of differentially expressed miRNAs. Two miRNA expression profiles (GSE90603 and GSE103229) were obtained from the GEO database. GSE90603 contained 16 GBM and 7 normal tissue samples, and GSE103229 contained 5 GBM and 5 normal tissue samples. A total of 64 differentially expressed miRNAs were identified in the GSE90603 dataset, and a total of 48 were found in GSE103229. Twelve differentially expressed miRNAs were observed to overlap between these two datasets (Fig. 1), and the analysis results are presented in Table I. 


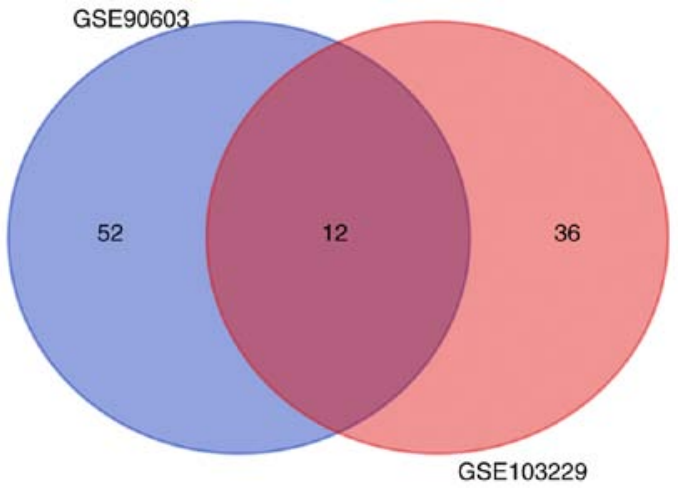

Figure 1. Differentially expressed miRNAs in GSE90603 and GSE103229 databases. A total of 64 differentially expressed miRNAs were identified in GSE90603, while 48 were found in GSE103229. There were 12 overlapping differentially expressed miRNAs. miRNAs, microRNAs.

Kaplan-Meier survival curves of 12 differentially expressed miRNAs. The overall cancer survival was predicted using OncomiR, and the results were presented with a Kaplan-Meier survival curve. In the present study, the glioma patients were divided into high-risk and low-risk cohorts according to the expression levels of the selected miRNAs. The survival analysis results suggested that only three miRNAs reached significant levels among the 12 differentially expressed miRNAs when predicting overall cancer survival, namely hsa-miR-7-5p, hsa-miR-21-3p and hsa-miR-455-3p (Fig. 2). The clinical value of miR-455-3p in glioma has not been reported in any previous studies and its differential expression level was the most significant (Table I).

Upregulation of miR-455-3p in glioma tissues. The expression levels of miR-455-3p were examined in 108 glioma and 95 normal brain tissue samples. The RT-qPCR results suggested that the expression of miR-455-3p was significantly upregulated in glioma tissues compared with normal tissues $(\mathrm{P}<0.001$; Fig. 3).

Association of miR-455-3p expression with clinicopathological features of patients with glioma. The clinical data of 108 patients with glioma are presented in Table II. The mean expression level of miR-455-3p in glioma tissues was identified as a cut-off level.

The patients were divided into low $(n=44)$ and high miR-455-3p expression $(n=64)$ groups. Differences between groups were compared using the $\chi^{2}$ test. It was observed that the upregulation of miR-455-3p expression demonstrated significant association with high WHO grade $(\mathrm{P}=0.006)$ and low KPS, $\mathrm{P}=0.037$ ) in glioma patients. However, miR-455-3p expression was not significantly associated with other clinicopathological features including age, sex and tumor size (all P>0.05; Table II).

Prognostic value of miR-455-3p expression in patients with glioma. The Kaplan-Meier survival curve and the log-rank test were performed to evaluate the association between miR-455-3p expression and patient survival. It was identified that patients with high miR-455-3p expression had significantly lower 5-year overall survival than those with low miR-455-3p
Table I. Twelve differentially expressed miRNAs in the overlap of GSE90603 and GSE103229 datasets.

\begin{tabular}{lll}
\hline miRNA_ID & adj.P.Val & Log FC \\
\hline hsa-miR-455-3p & $2.03 \times 10^{-6}$ & 2.30159 \\
hsa-miR-218-5p & $1.95 \times 10^{-4}$ & -2.90172 \\
hsa-miR-7-5p & $3.69 \times 10^{-4}$ & -3.25590 \\
hsa-miR-139-5p & $6.13 \times 10^{-4}$ & -2.88092 \\
hsa-miR-129-1-3p & $6.46 \times 10^{-4}$ & -2.13994 \\
hsa-miR-3200-3p & $9.75 \times 10^{-4}$ & -2.00359 \\
hsa-miR-124-3p & $1.43 \times 10^{-3}$ & -4.08201 \\
hsa-miR-129-5p & $2.31 \times 10^{-3}$ & -2.68730 \\
hsa-miR-21-3p & $3.74 \times 10^{-3}$ & 2.308139 \\
hsa-miR-338-5p & $4.85 \times 10^{-3}$ & -2.08672 \\
hsa-miR-138-5p & $6.96 \times 10^{-3}$ & -3.01343 \\
hsa-miR-491-5p & $9.93 \times 10^{-3}$ & -2.03053 \\
\hline
\end{tabular}

miRNA, microRNA; adj.P.Val, adjusted P-value; FC, fold change.

expression (log-rank $\mathrm{P}=0.001$; Fig. 4). The multivariate Cox regression analysis further suggested that miR-455-3p (hazard ratio=2.136; 95\% $\mathrm{CI}=1.177-3.877 ; \mathrm{P}=0.013$ ) was an independent prognostic indicator for overall survival in patients with glioma (Table III).

\section{Discussion}

Glioma is an aggressive central nervous system malignancy, which greatly affects human health (16). Owing to rapid proliferation, metastasis and angiogenesis, this disease is relatively hard to treat $(17,18)$. The prognosis of patients with glioma is relatively poor due to metastasis and recurrence $(3,19)$. The incidence and mortality rates of glioma are continuously increasing (1). Although numerous epigenetic changes have been identified to be associated with glioma, the pathogenesis is not fully understood. A recent study reported that a number of abnormal miRNAs were found to be involved in the transcriptional regulatory network associated with tumor initiation and progression (20). Therefore, systematic and integrative analysis is required to explore key miRNAs that may contribute to tumor progression.

Currently, microarray and bioinformatics analyses are widely used to investigate potential targets for the diagnosis and therapy of different types of human cancer $(21,22)$. In the present study, a total of 64 differentially expressed miRNAs were identified in GSE90603, and 48 were found in GSE103229. Among them, 12 differentially expressed miRNAs were observed to overlap between these two datasets. OncomiR is an online resource for identifying aberrantly expressed miRNAs in cancer. In the present study, OncomiR was used to examine the predictive value of the differentially expressed miRNAs in the overall survival rate of patients with glioma. Kaplan-Meier survival curves were generated and the survival analysis suggested that only three miRNAs were significantly upregulated among the 12 differentially expressed miRNAs, namely hsa-miR-7-5p, hsa-miR-21-3p and hsa-miR-455-3p. 

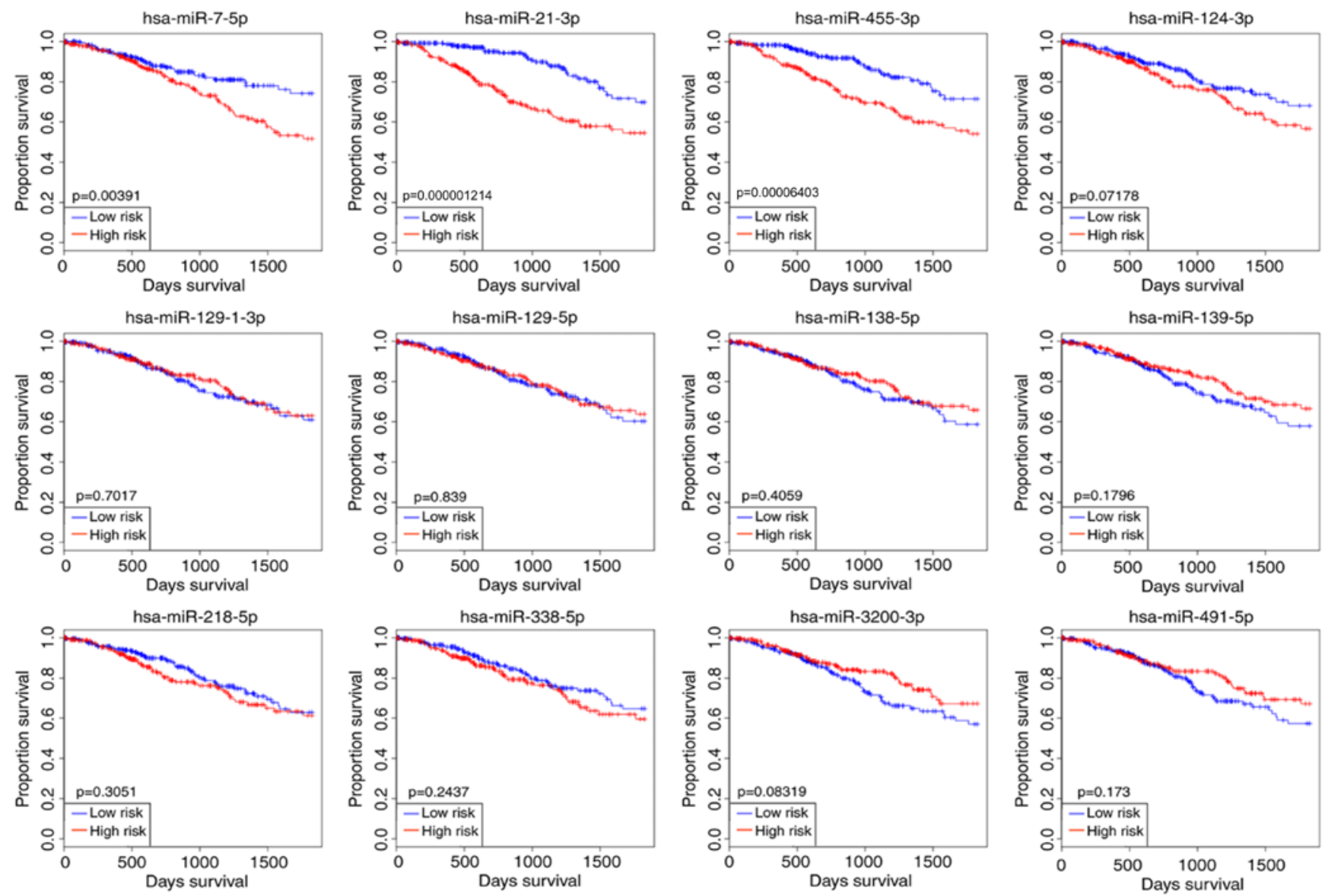

Figure 2. Survival analysis of the 12 identified differentially expressed miRNAs. The glioma patients were divided into high-risk cohort low-risk groups according to the miRNA expression levels. OncomiR was used to examine the significance of the observed differentially expressed miRNAs in predicting overall cancer survival, and only three miRNAs were significantly upregulated among the 12 differentially expressed miRNAs, namely hsa-miR-7-5p, hsa-miR-21-3p and hsa-miR-455-3p $(\mathrm{P}<0.05)$. miRNAs/miRs, microRNAs.

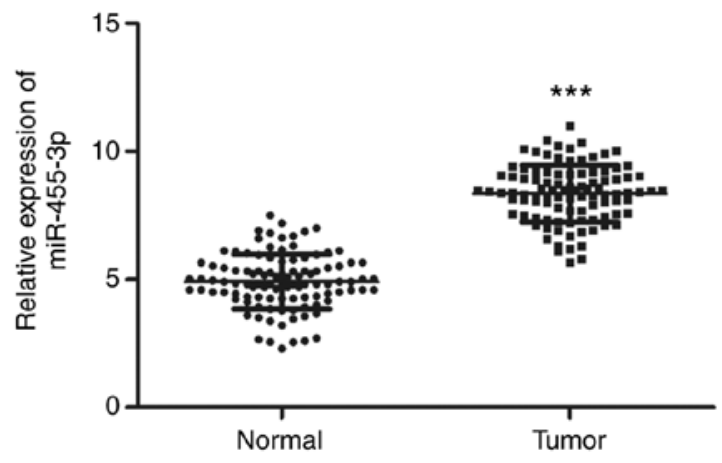

Figure 3. Expression levels of miR-455-3p measured by RT-qPCR in glioma and normal brain tissues. miR-455-3p expression was significantly upregulated in the glioma tissues compared with the normal brain tissues. ${ }^{* * * *} \mathrm{P}<0.001$. RT-qPCR, reverse transcription-quantitative polymerase chain reaction; $\mathrm{miR}$, microRNA

All results indicated the potential involvement of miR-7-5p, miR-21-3p and miR-455-3p in glioma. Regarding miR-7-5p, Liu et al (23) have reported its downregulation in GBM microvessels compared with normal brain capillaries. In the same study, it was demonstrated that miR-7-5p may function as a tumor suppressor by inhibiting vascular endothelial cell proliferation through Raf1 targeting. Furthermore, different expression of miR-7-5p has been detected in other types of human cancer, including bladder cancer, breast cancer and melanoma (24-26). These findings indicate the crucial role of miR-7-5p in cancer development. miR-21-3p, the passenger strand of pre-miR-21, has been widely reported to be aberrantly expressed in various types of human cancer. Tseng et al (27) suggested that miR-21-3p expression is significantly upregulated in oral squamous cell carcinoma tissues compared with corresponding adjacent normal tissues. In glioma, miR-21-3p was proven to regulate glioma cell proliferation and apoptosis by downregulating the expression of PTEN protein (28).

Regarding miR-455-3p, it has been reported to serve a role in acquired temozolomide resistance in patients with glioma (29-31). A recent study suggested that miR-455-3p was significantly increased in glioma cell lines and may regulate tumor cell progression (32). However, its expression levels and clinical significance have not been assessed in patients with glioma. In the present study, the bioinformatics analysis results suggested that the differential expression level of miR-455-3p in glioma was the most significant. Subsequently, its expression in patients with glioma was examined. Consistent with the bioinformatics analysis results, the expression levels of miR-455-3p were significantly upregulated in glioma tissues compared with normal tissues. Based on malignancy degree, WHO has divided gliomas into four grades: i) Pilocytic astrocytoma (WHO grade I), ii) diffuse astrocytoma (WHO 
Table II. Association of miR-455-3p with the clinicopathological features of patients with glioma.

\begin{tabular}{|c|c|c|c|c|}
\hline \multirow[b]{2}{*}{ Feature } & \multirow[b]{2}{*}{$\mathrm{n}=108$} & \multicolumn{2}{|c|}{ miR-455-3p expression } & \multirow[b]{2}{*}{ P-value } \\
\hline & & Low $(n=44)$ & High $(n=64)$ & \\
\hline Age (years) & & & & 0.383 \\
\hline$\leq 60$ & 51 & 23 & 28 & \\
\hline$>60$ & 57 & 21 & 36 & \\
\hline Sex & & & & 0.942 \\
\hline Male & 56 & 23 & 33 & \\
\hline Female & 52 & 21 & 31 & \\
\hline Tumor size (cm) & & & & 0.354 \\
\hline$\geq 5.0$ & 67 & 25 & 42 & \\
\hline$<5.0$ & 41 & 19 & 22 & \\
\hline WHO grade & & & & $0.006^{\mathrm{a}}$ \\
\hline I-II & 64 & 33 & 31 & \\
\hline III-IV & 44 & 11 & 33 & \\
\hline KPS & & & & $0.037^{\mathrm{b}}$ \\
\hline$<80$ & 62 & 20 & 42 & \\
\hline$\geq 80$ & 46 & 24 & 22 & \\
\hline
\end{tabular}

${ }^{\mathrm{a}} \mathrm{P}<0.01 .{ }^{\mathrm{b}} \mathrm{P}<0.05$. WHO, World Health Organization; KPS, Karnofsky performance scale.

grade II), iii) anaplastic astrocytoma (WHO grade III); and iv) GBM multiform (WHO grade IV) (33). In the present study, the histopathological WHO grades and KPS score, which are currently used to predict prognosis in patients with glioma, were recorded (34). It was observed that the upregulation of miR-455-3p was closely associated with high WHO grade and low KPS score, indicating the potential prognostic value of miR-455-3p in glioma. As suggested by the bioinformatics analysis, miR-455-3p may be used as a potential prognostic marker in glioma. Moreover, the Kaplan-Meier survival curves and log-rank test were also performed, and it was confirmed that miR-455-3p served as an independent prognostic indicator for overall survival and that high miR-455-3p expression levels were associated with poor prognosis. The abnormal expression of miR-455-3p and its prognostic value have been widely reported in numerous types of human cancer (35-37). For example, Gao et al (37) reported that miR-455-3p was significantly downregulated in non-small cell lung cancer, while low miR-455-3p expression was an unfavorable prognostic factor for overall survival. Another study suggested that miR-455-3p functioned as a tumor suppressor in esophageal squamous cell carcinoma (ESCC) and inhibited cell proliferation and invasion by targeting FAM83F. In addition, low miR-455-3p expression was identified as an unfavorable prognostic factor for the overall survival of patients with ESCC (38). Nevertheless, the functional role and underlying mechanism of miR-455-3p in glioma was not examined in the present study, thus further studies are required. Additionally, certain limitations are included in the present study. Firstly, it is generally accepted
Table III. Multivariate Cox regression analysis for miR-455-3p in patients with glioma.

\begin{tabular}{lccc}
\hline & \multicolumn{2}{c}{ Multivariate analysis } & \\
\cline { 2 - 3 } Variable & HR & $95 \% \mathrm{CI}$ & P-value \\
\hline miR-455-3p & 2.136 & $1.177-3.877$ & $0.013^{\mathrm{a}}$ \\
Age & 0.899 & $0.551-1.468$ & 0.671 \\
Sex & 1.115 & $0.693-1.793$ & 0.654 \\
Tumor size & 0.808 & $0.490-1.332$ & 0.403 \\
WHO grade & 1.607 & $0.973-2.654$ & 0.064 \\
KPS & 0.789 & $0.472-1.319$ & 0.367 \\
\hline
\end{tabular}

${ }^{\mathrm{a}} \mathrm{P}<0.05$. miR, microRNA; HR, hazard ratio; $\mathrm{CI}$, confidence interval; WHO, World Health Organization; KPS, Karnofsky performance scale.

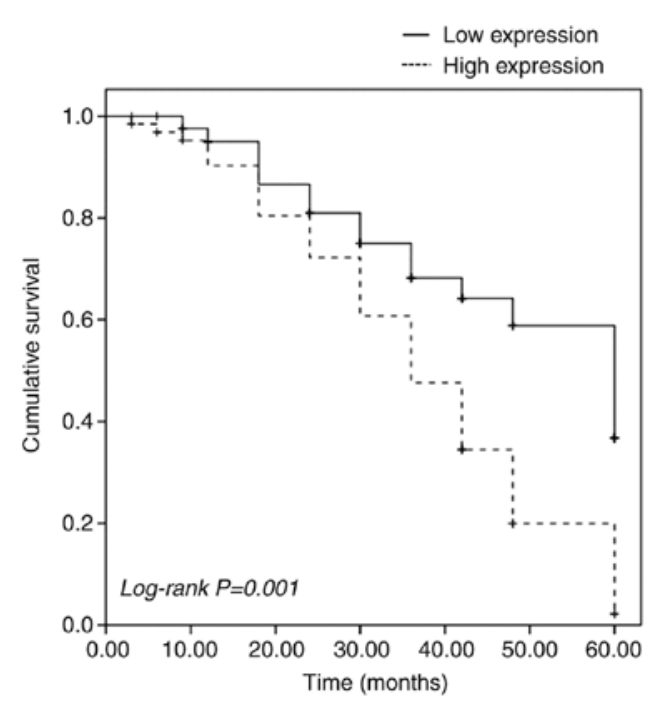

Figure 4. Kaplan-Meier survival analysis for the patients with glioma based on the expression of miR-455-3p. Patients with high miR-455-3p expression had shorter survival time than those with low miR-455-3p expression (log-rank $\mathrm{P}=0.001)$. miR, microRNA.

that higher tumor grade is associated with poorer prognosis, however KPS revealed no significant association with the prognosis of patients with glioma, although results indicated that the difference was close to statistical significance. This may be due to the sample size being relatively small; a larger study would be required to confirm the present results of the present study. Secondly, according to the bioinformatics analysis, several differentially expressed miRNAs were identified to be associated with glioma prognosis, though only one major miRNA was investigated. Additional miRNAs may be investigated in future studies. Lastly, the clinical and pathological information included in the present study were limited. For example, although the mutation status of isocitrate dehydrogenase 1 and $1 \mathrm{p} 19 \mathrm{q}$, which may serve an important role in the prognosis of patients with glioma, have attracted broad attention during the last decades $(32,39)$; these were not examined in the present study. In future studies, these clinical and pathological parameters should be included. 
In conclusion, 12 differentially expressed miRNAs were identified via integrative analysis of GEO data and OncomiR analysis suggested that among them, three miRNAs were able to accurately predict overall cancer survival in glioma. Furthermore, miR-455-3p upregulation was validated in patients with glioma, and it was indicated as a potential prognostic biomarker for glioma. The present study identified a series of miRNAs with potential functional roles in the pathogenesis of glioma, and provides evidence for the use of miR-455-3p as a promising biomarker for glioma prognosis.

\section{Acknowledgements}

Not applicable.

\section{Funding}

The present study was funded by the Natural Science Foundation of Fujian Province (grant nos. 2018Y0067 and 2017J0105), the Startup Fund for scientific research of Fujian Medical University (grant no. 2017XQ2049), 900th Hospital of the Joint Logistics Team Innovation Team Fund (grant nos. 2014CXTD07 and 2018Z03) and the Fujian Science and Technology Plan Key Project (grant no. 2014Y0036).

\section{Availability of data and materials}

All data generated or analyzed during this study are included in this published article.

\section{Authors' contributions}

WW and SM conceived and designed the study, acquired the data and drafted the manuscript. QZ was involved in drafting the manuscript and data analysis. LX and SW were responsible for data interpretation and critically revised the manuscript. All authors read and approved the final manuscript.

\section{Ethics approval and consent to participate}

All procedures of the present study were reviewed and approved by the Ethics Committee of The 900th Hospital of the Joint Logistics Team. Written informed consent was obtained from each participant.

\section{Patient consent for publication}

Not applicable.

\section{Competing interests}

The authors declare that they have no competing interests.

\section{References}

1. Yang D, Yuan Y, Zhang S, Zhao K, Li F, Ren H, Zhang Z and Yu Y: Association between IL-13 Gene rs20541 polymorphism and glioma susceptibility: A meta-analysis. Oncol Res Treat 41: 14-21, 2018.
2. Bas Ayata H, Ceylan C, Kilic A, Guden M and Engin K: Comparison of multiple treatment planning techniques for high-grade glioma tumors near to critical organs. Oncol Res Treat 41: 514-519, 2018.

3. Hua S, Li H, Liu Y, Zhang J, Cheng Y and Dai C: High expression of GALNT7 promotes invasion and proliferation of glioma cells. Oncol Lett 16: 6307-6314, 2018.

4. Su C, Li H and Gao W: TRIM28 is overexpressed in glioma and associated with tumor progression. Onco Targets Ther 11: 6447-6458, 2018

5. Lai X, Deng Z, Guo H, Zhu X and Tu W: HP1 $\alpha$ is highly expressed in glioma cells and facilitates cell proliferation and survival. Cancer Biomark 20: 453-460, 2017.

6. Paul S: Integration of miRNA and mRNA expression data for understanding etiology of gynecologic cancers. Methods Mol Biol 1912: 323-338, 2019.

7. Wang S, Chen Y, Yu X, Lu Y, Wang $\mathrm{H}$, Wu F and Teng L: miR-129-5p attenuates cell proliferation and epithelial mesenchymal transition via HMGB1 in gastric cancer. Pathol Res Pract 215: 676-682, 2019.

8. Huang X, Liang M, Dittmar R and Wang L: Extracellular microRNAs in urologic malignancies: Chances and challenges. Int J Mol Sci 14: 14785-14799, 2013.

9. Zhang JF, Zhang JS, Zhao ZH, Yang PB, Ji SF, Li N, Shi QD, Tan J, Xu X, Xu CB and Zhao LY: MicroRNA-770 affects proliferation and cell cycle transition by directly targeting CDK8 in glioma. Cancer Cell Int 18: 195, 2018.

10. Lu GF, Geng F, Xiao Z, Chen YS, Han Y, You CY, Gong NL, Xie ZM and Pan M: MicroRNA-6807-3p promotes the tumorigenesis of glioma by targeting downstream DACH1. Brain Res 1708: 47-57, 2019.

11. Tian W, Wu W, Li X, Rui X and Wu Y: MiRNA-139-3p inhibits the proliferation, invasion, and migration of human glioma cells by targeting MDA-9/syntenin. Biochem Biophys Res Commun 508: 295-301, 2019.

12. Gulluoglu S, Tuysuz EC, Sahin M, Kuskucu A, Kaan Yaltirik C, Ture U, Kucukkaraduman B, Akbar MW, Gure AO, Bayrak OF and Dalan AB: Simultaneous miRNA and mRNA transcriptome profiling of glioblastoma samples reveals a novel set of OncomiR candidates and their target genes. Brain Res 1700: 199-210, 2018.

13. Zhu J, Ye J, Zhang L, Xia L, Hu H, Jiang H, Wan Z, Sheng F, Ma Y, Li W, et al: Differential expression of circular RNAs in glioblastoma multiforme and its correlation with prognosis. Transl Oncol 10: 271-279, 2017.

14. Wong NW, Chen Y, Chen S and Wang X: OncomiR: An online resource for exploring pan-cancer microRNA dysregulation. Bioinformatics 34: 713-715, 2018.

15. Livak KJ and Schmittgen TD: Analysis of relative gene expression data using real-time quantitative PCR and the 2(-Delta Delta $\mathrm{C}(\mathrm{T}))$ method. Methods 25: 402-408, 2001.

16. Huang Q, Wang C, Hou Z, Wang G, Lv J, Wang H, Yang J, Zhang $Z$ and Zhang H: Serum microRNA-376 family as diagnostic and prognostic markers in human gliomas. Cancer Biomark 19: 137-144, 2017.

17. Wang $X$, Chen $X$, Sun L, Bi X, He H, Chen L and Pang J: The function of MMP-28/TGF- $\beta$ induced cell apoptosis in human glioma cells. Exp Ther Med 16: 2867-2874, 2018.

18. Xu L, Yu QW, Fang SQ, Zheng YK and Qi JC: MiR-650 inhibits the progression of glioma by targeting FAM83F. Eur Rev Med Pharmacol Sci 22: 8391-8398, 2018.

19. Hu S, Xu L, Li L, Luo D, Zhao H, Li D and Peng B: Overexpression of lncRNA PTENP1 suppresses glioma cell proliferation and metastasis in vitro. Onco Targets Ther 12: 147-156, 2019.

20. Xie C, Xu M, Lu D, Zhang W, Wang L, Wang H, Li J, Ren F and Wang C: Candidate genes and microRNAs for glioma pathogenesis and prognosis based on gene expression profiles. Mol Med Rep 18: 2715-2723, 2018.

21. Long T, Liu Z, Zhou X, Yu S, Tian H and Bao Y: Identification of differentially expressed genes and enriched pathways in lung cancer using bioinformatics analysis. Mol Med Rep 19: 2029-2040, 2019

22. Rahman MR, Islam T, Gov E, Turanli B, Gulfidan G, Shahjaman M, Banu NA, Mollah MNH, Arga KY and Moni MA: Identification of prognostic biomarker signatures and candidate drugs in colorectal cancer: Insights from systems biology Analysis. Medicina (Kaunas) 55: pii: E20, 2019.

23. Liu Z, Liu Y, Li L, Xu Z, Bi B, Wang Y and Li JY: MiR-7-5p is frequently downregulated in glioblastoma microvasculature and inhibits vascular endothelial cell proliferation by targeting RAF1. Tumour Biol 35: 10177-10184, 2014. 
24. Li J, Qiu M, An Y,Huang J and Gong C: miR-7-5p acts as a tumor suppressor in bladder cancer by regulating the hedgehog pathway factor Gli3. Biochem Biophys Res Commun 503: 2101-2107, 2018.

25. Block I, Burton M, Sorensen KP, Andersen L, Larsen MJ, Bak M, Cold S, Thomassen M, Tan Q and Kruse TA: Association of miR-548c-5p, miR-7-5p, miR-210-3p, miR-128-3p with recurrence in systemically untreated breast cancer. Oncotarget 9: 9030-9042, 2018.

26. Giles KM, Brown RA, Epis MR, Kalinowski FC and Leedman PJ: miRNA-7-5p inhibits melanoma cell migration and invasion. Biochem Biophys Res Commun 430: 706-710, 2013.

27. Tseng HH, Tseng YK, You JJ, Kang BH, Wang TH, Yang CM, Chen HC, Liou HH, Liu PF, Ger LP and Tsai KW: Next-generation sequencing for microRNA profiling: MicroRNA-21-3p promotes oral cancer metastasis. Anticancer Res 37: 1059-1066, 2017.

28. Li SJ, Zhou J, Zhang L, Xiang W, Hu Q, He YY and Chen LG: The effect of miR-21 on SWOZ2 glioma cells and its biological mechanism. J BUON 22: 468-473, 2017.

29. Ujifuku K, Mitsutake N, Takakura S, Matsuse M, Saenko V, Suzuki K, Hayashi K, Matsuo T, Kamada K, Nagata I and Yamashita S: miR-195, miR-455-3p and miR-10a $\left({ }^{*}\right)$ are implicated in acquired temozolomide resistance in glioblastoma multiforme cells. Cancer Lett 296: 241-248, 2010.

30. Mizoguchi M, Guan Y, Yoshimoto K, Hata N, Amano T, Nakamizo A and Sasaki T: Clinical implications of microRNAs in human glioblastoma. Front Oncol 3: 19, 2013.

31. Zhang Y, Dutta A and Abounader R: The role of microRNAs in glioma initiation and progression. Front Biosci (Landmark Ed) 17: 700-712, 2012.

32. Han J, Xiong Y, Deng H, Zhou J, Peng L, Xiang W, Ming Y and Chen L: MiR-455-3p regulates glioma cell proliferation by targeting PAX6. Tropical J Pharmaceutical Res 18: 689-695, 2019.
33. Louis DN, Ohgaki H, Wiestler OD, Cavenee WK, Burger PC, Jouvet A, Scheithauer BW and Kleihues P: The 2007 WHO classification of tumours of the central nervous system. Acta Neuropathol 114: 97-109, 2007.

34. Zhang W, Zhao W, Ge C, Li X, Yang X, Xiang Y and Sun Z: Decreased let-7b is associated with poor prognosis in glioma. Medicine (Baltimore) 98: e15784, 2019.

35. Guo J, Liu C, Wang W, Liu Y, He H, Chen C, Xiang R and Luo Y: Identification of serum miR-1915-3p and miR-455-3p as biomarkers for breast cancer. PLoS One 13: e0200716, 2018.

36. Chai L, Kang XJ, Sun ZZ, Zeng MF, Yu SR, Ding Y, Liang JQ, Li TT and Zhao J: MiR-497-5p, miR-195-5p and miR-455-3p function as tumor suppressors by targeting hTERT in melanoma A375 cells. Cancer Manag Res 10: 989-1003, 2018.

37. Gao X, Zhao H, Diao C, Wang X, Xie Y, Liu Y, Han J and Zhang M: miR-455-3p serves as prognostic factor and regulates the proliferation and migration of non-small cell lung cancer through targeting HOXB5. Biochem Biophys Res Commun 495: 1074-1080, 2018

38. Yang H, Wei YN, Zhou J, Hao TT and Liu XL: MiR-455-3p acts as a prognostic marker and inhibits the proliferation and invasion of esophageal squamous cell carcinoma by targeting FAM83F. Eur Rev Med Pharmacol Sci 21: 3200-3206, 2017.

39. Chen H, Judkins J, Thomas C, Wu M, Khoury L, Benjamin CG, Pacione D, Golfinos JG, Kumthekar P, Ghamsari F, et al: Mutant IDH1 and seizures in patients with glioma. Neurology 88: 1805-1813, 2017

This work is licensed under a Creative Commons Attribution-NonCommercial-NoDerivatives 4.0 International (CC BY-NC-ND 4.0) License. 